\title{
Quantum signatures of an oscillatory instability in the Bose-Hubbard trimer
}

Peter Jason, Magnus Johansson and Katarina Kirr

\section{Linköping University Post Print}

N.B.: When citing this work, cite the original article.

Original Publication:

Peter Jason, Magnus Johansson and Katarina Kirr, Quantum signatures of an oscillatory instability in the Bose-Hubbard trimer, 2012, Physical Review E. Statistical, Nonlinear, and Soft Matter Physics, (86), 1, 016214.

http://dx.doi.org/10.1103/PhysRevE.86.016214

Copyright: American Physical Society

http://www.aps.org/

Postprint available at: Linköping University Electronic Press

http://urn.kb.se/resolve?urn=urn:nbn:se:liu:diva-79982 


\title{
Quantum signatures of an oscillatory instability in the Bose-Hubbard trimer
}

\author{
Peter Jason, ${ }^{1}$ Magnus Johansson, ${ }^{1, *}$ and Katarina Kirr ${ }^{1,2}$ \\ ${ }^{1}$ Department of Physics, Chemistry and Biology (IFM), Linköping University, SE-581 83 Linköping, Sweden \\ ${ }^{2}$ Institute of Electrophysics and Radiation Technologies, 28 Chernyshevskoho Street, 61002 Kharkiv, Ukraine
}

(Received 5 April 2012; published 17 July 2012)

\begin{abstract}
We study the Bose-Hubbard model for three sites in a symmetric, triangular configuration and search for quantum signatures of the classical regime of oscillatory instabilities, known to appear through Hamiltonian Hopf bifurcations for the "single-depleted-well" family of stationary states in the discrete nonlinear Schrödinger equation. In the regimes of classical stability, single quantum eigenstates with properties analogous to those of the classical stationary states can be identified already for rather small particle numbers. On the other hand, in the instability regime the interaction with other eigenstates through avoided crossings leads to strong mixing, and no single eigenstate with classical-like properties can be seen. We compare the quantum dynamics resulting from initial conditions taken as perturbed quantum eigenstates and SU(3) coherent states, respectively, in a quantum-semiclassical transitional regime of 10-100 particles. While the perturbed quantum eigenstates do not show a classical-like behavior in the instability regime, a coherent state behaves analogously to a perturbed classical stationary state, and exhibits initially resonant oscillations with oscillation frequencies well described by classical internal-mode oscillations.
\end{abstract}

DOI: 10.1103/PhysRevE.86.016214

PACS number(s): 05.45.Mt, 03.65.Sq, 03.75.Kk, 03.75.Lm

\section{INTRODUCTION}

Oscillatory instabilities, where a small perturbation of a stationary (or time-periodic) state yields an initial oscillatory dynamics with exponentially increasing amplitude, are quite ubiquitous in the classical dynamics of Hamiltonian nonlinear lattices (see, e.g., [1] and references therein). They typically appear for states with inhomogeneous amplitude distribution, dividing the lattice into sublattices of sites with small and large amplitudes, respectively, as a result of resonances between internal oscillations from the different sublattices. In the linear stability problem, oscillatory instabilities arise through Hamiltonian Hopf bifurcations yielding eigenvalues with nonzero real as well as imaginary parts. Well-known examples for one-dimensional lattices are, e.g., two-site localized "twisted" modes [2,3], discrete dark solitons ("dark breathers") [4,5], spatially periodic or quasiperiodic nonlinear standing waves [6], and gap modes in diatomic chains [7,8]. In the latter contexts, oscillatory instabilities may play an important role in the initial stages of breather formation and thermalization processes $[6,8]$.

Arguably the simplest example of an oscillatory instability appears in the symmetric discrete nonlinear Schrödinger (DNLS) trimer, with $f=3$ degrees of freedom interacting identically with each other [9]. (The DNLS dimer, $f=2$, is integrable and its stationary solutions may exhibit only nonoscillatory instabilities [9].) As first discussed in [9], one particular family of exact stationary solutions, corresponding to two sites in antiphase oscillations and the third site identically zero, is oscillatorily unstable in an interval of intermediate nonlinearity. Such solutions were later termed "single depleted well" (SDW) states [10]. The unstable dynamics, investigated in some detail in [1], involves a resonant oscillation between one mode whose main effect is to populate the empty site and another mode corresponding

\footnotetext{
*mjn@ifm.liu.se; URL: https://people.ifm.liu.se/majoh
}

mainly to internal population oscillations between the two nonzero sites.

The DNLS model is a classical limit of the well-known Bose-Hubbard model for interacting bosons on a lattice (therefore also termed the "quantum DNLS" model [11]), when the total number of bosons $N$ goes to infinity. In particular, the trimer has a direct physical application for a Bose-Einstein condensate in symmetric triple-well traps; see, e.g., [12-14] for some proposed realizations. The natural question then arises: how can an oscillatory instability appear in the classical limit (" $\hbar \rightarrow 0$ ") of a fundamental quantum lattice model?

One may consider several possible ways to characterize the appearance of the classical oscillatory instability from the quantum problem, and it is the purpose of the present work to discuss and compare some different approaches, mainly through numerical computations for modest particle numbers. We will characterize certain quantum signatures of the classical instability transition, which may be experimentally observable as the number of bosons per lattice site increases.

First, we discuss the energy spectra and eigenstates for particle number $N$ increasing from 10 to 90 . In the stronginteraction or weak-coupling ("anticontinuous") limit there are, for each finite (even) $N$, three degenerate quantum eigenstates of the form $[N / 2, N / 2,0]$, with exactly zero population at one of the sites. Following these states towards weaker interaction or larger coupling, they start to mix strongly with other states through avoided crossings in the spectrum (as seen already in [15]), and it is not a priori evident which of the quantum states (if any) should be considered as a quantum counterpart to a classical single-depleted-well state. We will discuss various ways to characterize a "good" quantum SDW eigenstate and show that such typical "goodness" measures drastically decrease in an intermediate-parameter regime dominated by strong quantum resonances, which approaches the regime of classical oscillatory instability as the particle number increases. 
We then go on to investigate the quantum dynamics for slightly perturbed quantum SDW-like eigenstates, and find that such perturbations in general are not able to reproduce a classical-like behavior in the instability regime.

On the other hand, it is well known (see, e.g., [12-14,16,17]) that for each finite $N$, one may construct $\mathrm{SU}(f)$ coherent states ( $f=3$ for a trimer), for which the time evolution obtained from the Bose-Hubbard model exactly reproduces the classical DNLS dynamics in the limit $N \rightarrow \infty$. [The $\mathrm{SU}(f)$ coherent states are equivalent to the Hartree states of [18], but differ from the Glauber coherent states used, e.g., in $[19,20]$ essentially in that the former conserve total particle number for any $N$, while the latter conserve the rescaled particle number, the DNLS norm, only in the classical limit $N \rightarrow \infty$.] However, even if the classical solution is an exact stationary solution (the absolute values of the coherent-state parameters are time independent), the corresponding coherent state will generally not be an exact quantum eigenstate for any finite $N$.

Thus, since the coherent states are the most "classicallike" states but generally not eigenstates, one may look for signatures in the properties of the finite- $N$ coherent states rather than the eigenstates. This is the approach used, e.g., in [14], and may be useful in particular for large $N$. As we shall see, for $N$ approaching 100 it is indeed possible to trace the classical resonant oscillations in pure quantum dynamics using SU(3) coherent states as initial conditions, although the quantum instability transition for such relatively small values of $N$ becomes smooth rather than sharp as in the classical model.

Although, to the best of our knowledge, quantum signatures of the classical Hamiltonian Hopf bifurcations and oscillatory instabilities of stationary, non-current-carrying solutions have not been analyzed before, there are several earlier works discussing quantum counterparts to other types of classical DNLS dynamical instability transitions that deserve to be mentioned in this context. For example, the classical modulational instability threshold of current-carrying constantamplitude lattice waves [21] was analyzed from the quantum Bose-Hubbard perspective in [22], and in particular it was found that quantum fluctuations may lead to a substantial broadening of the classical transition for a one-dimensional lattice (as we will find also for our case). Another well-studied example is the classical self-trapping transition, where, for an attractive effective interaction, the delocalized ground state becomes unstable and a stable localized ground state appears in a bifurcation [9]. The quantization of this transition, which appears already for the dimer case $f=2$, has been analyzed in a large number of papers starting from [18,23], and very recently in [24] (to which we also refer for a more complete set of references on this issue), where a major result is that, again, quantum fluctuations result in a critical regime rather than a single bifurcation point. We should, however, stress that both these instability transitions appear for perturbed ground states (or highest-energy states, depending on whether the interaction is repulsive or attractive), while Hamiltonian Hopf bifurcations necessarily must appear for excited states since the two resonating internal modes must contribute with different signs to the total energy of the stationary state (see, e.g., [1]). Thus, the physical origin of the instabilities studied here is fundamentally different from that in earlier works.

\section{MODEL}

The form of the Bose-Hubbard model that we will use is

$$
\hat{H}_{\mathrm{BH}}=\sum_{i=1}^{3}\left\{\alpha \hat{a}_{i}^{\dagger} \hat{a}_{i}^{\dagger} \hat{a}_{i} \hat{a}_{i}+\hat{a}_{i}^{\dagger}\left(\hat{a}_{i+1}+\hat{a}_{i-1}\right)\right\},
$$

with periodic boundary conditions and nonlinear parameter $\alpha>0$. Using boson commutation relations $\left[\hat{a}_{i}, \hat{a}_{j}^{\dagger}\right]=\delta_{i, j}$ and the number operator $\hat{n}_{i}=\hat{a}_{i}^{\dagger} \hat{a}_{i}$, this can also be written as

$$
\hat{H}_{\mathrm{BH}}=\sum_{i=1}^{3}\left\{\alpha \hat{n}_{i}\left(\hat{n}_{i}-1\right)+\hat{a}_{i}^{\dagger}\left(\hat{a}_{i+1}+\hat{a}_{i-1}\right)\right\} .
$$

Following [12-14,16-18] and making an ansatz of the wave function as an $\mathrm{SU}(3)$ coherent state with a given $N=$ $\sum_{i=1}^{3}\left\langle\hat{n}_{i}\right\rangle$, we obtain a dynamical equation for the coherentstate parameters $a_{i}$, which is the DNLS equation corresponding to the following effective classical Hamiltonian:

$\mathcal{H}=\left\langle\hat{H}_{\mathrm{BH}}\right\rangle=\sum_{i=1}^{3}\left\{\alpha \frac{(N-1)}{N}\left|a_{i}\right|^{4}+a_{i}^{*}\left(a_{i+1}+a_{i-1}\right)\right\}$,

with $\sum_{i=1}^{3}\left|a_{i}\right|^{2}=N$. Comparing with the notation in [1], identifying $C=\frac{1}{2 \alpha} \frac{N}{N-1}$ takes $-\frac{1}{2 \alpha} \frac{N}{N-1}(\mathcal{H}-2 N)$ into the classical Hamiltonian in Eq. (2) of [1].

Thus, from this identification, we can conclude that the classical condition [1,9] for oscillatory instability of the SDW stationary state $\left\{a_{i}\right\}=\{\sqrt{N / 2},-\sqrt{N / 2}, 0\}$, $9.077 \ldots<N / C<18$, translates for a quantum SU(3) coherent state into $4.5385 \ldots /(N-1)<\alpha<9 /(N-1)$. On the other hand, if one were to choose instead trial functions as tensor products of standard Glauber coherent states at each site, as is done, e.g., in [19,20] (eigenfunctions of the annihilation operators $\hat{a}_{i}$ at each site), one would again end up with a DNLS equation for the dynamics of these coherent state parameters, but corresponding to a classical Hamiltonian without the factor $(N-1) / N$ in (3). The above condition for oscillatory instability would then instead become $4.5385 \ldots / N<\alpha<$ $9 / N$. Clearly this distinction is irrelevant in the classical limit (assuming that $\alpha$ scales inversely with $N$ to have a finite energy per particle, $\mathcal{H} / N<\infty$, in this limit), and we have also found that the observed signatures of oscillatory instabilities for the pure quantum system (not too large $N$ ), described below, essentially cannot determine whether one coherent-state ansatz gives a more classical-like behavior than the other. Therefore, in the remainder of this paper we will show numerical results using, for convenience, $\alpha N$ rather than $\alpha(N-1)$ as parameter [as also in Sec. IV B where SU(3) coherent states are used as initial conditions].

Concerning the classical dynamics of perturbed SDW states in the unstable regime, it was discovered in [1] that a self-trapping transition appears at a critical value, $\alpha \approx$ $5.3 /(N-1)$ in the notation of (3). Below the transition, the unstable dynamics remains trapped close to the initial state due to phase-space-dividing Kolmogorov-Arnold-Moser (KAM) tori, so that, although the dynamics may be weakly 
chaotic, the amplitude of the initially unexcited site remains small forever. Above the transition the dynamics is strongly chaotic, and typically an intermittent population-inversion dynamics is observed with the small-amplitude oscillation moving chaotically between all three sites. As will be seen below, some traces of this transition can be identified also in the quantum model.

\section{ENERGY SPECTRUM AND EIGENSTATES}

We first illustrate in Fig. 1 an overall picture of the energy spectrum of Eq. (1) for particle numbers increasing from $N=10$ to $N=60$. (Pictures of the general trimer spectrum for particle numbers of this order were probably first shown in [15].)

Our initial strategy is to consider the family of exact eigenstates which approaches a state of the form $[N / 2, N / 2,0]$ for large $\alpha$. Due to translational invariance in the symmetric trimer configuration (periodic boundary conditions) there are three of these, one state with Bloch vector $k=0$ and two degenerate with $k= \pm 2 \pi / 3$; see, e.g., $[11,15]$. Explicitly, we may thus use the basis states

$$
\begin{aligned}
\left|n_{1}, n_{2}, n_{3}\right\rangle_{k} \equiv & \frac{1}{\sqrt{3}}\left(\left|n_{1}, n_{2}, n_{3}\right\rangle\right. \\
& \left.+e^{i k}\left|n_{3}, n_{1}, n_{2}\right\rangle+e^{2 i k}\left|n_{2}, n_{3}, n_{1}\right\rangle\right) .
\end{aligned}
$$

This introduces a tunneling time scale proportional to the inverse of the energy spacing between states with $k=0$ and $k= \pm 2 \pi / 3$ so that, close to the anticontinuous limit, an initially excited SDW state with the zero at a given site would in general not be an exact quantum eigenstate, but instead we should expect the "hole" to perform periodic quantum tunnelings between all three sites. Analogously as for single-quantum-breather states [18,25-27], we should expect this tunneling time scale to go to infinity as well in the anticontinuous as in the classical limits (properly rescaled). Thus, for a given (rescaled) nonlinearity strength where the SDW state is unstable, this should put a lower bound on the particle number for which quantum mechanics can properly reproduce the initial stages of an oscillatory instability: the tunneling frequency (energy splitting) must be small compared to the classical oscillation frequency of the unstable eigenmode. This will be discussed further in Sec. IV.
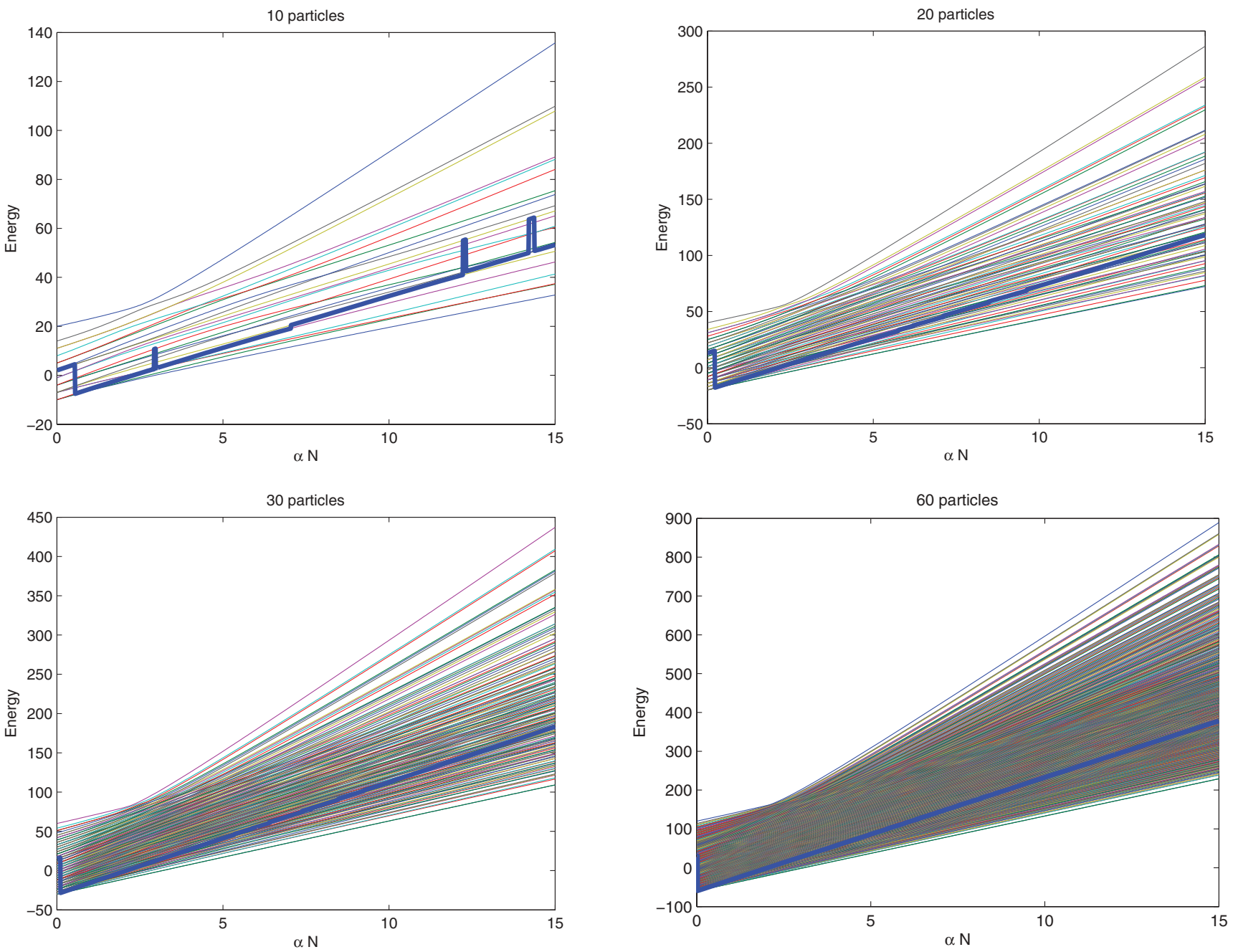

FIG. 1. (Color online) Energy spectrum of Eq. (1) vs $\alpha N$, for Bloch states with $k=0$ and $N=10,20,30,60$ particles, respectively. The state with the largest expansion coefficient for the basis function $|N / 2, N / 2,0\rangle_{0}$ (see text) is plotted with a bold (blue) line. 


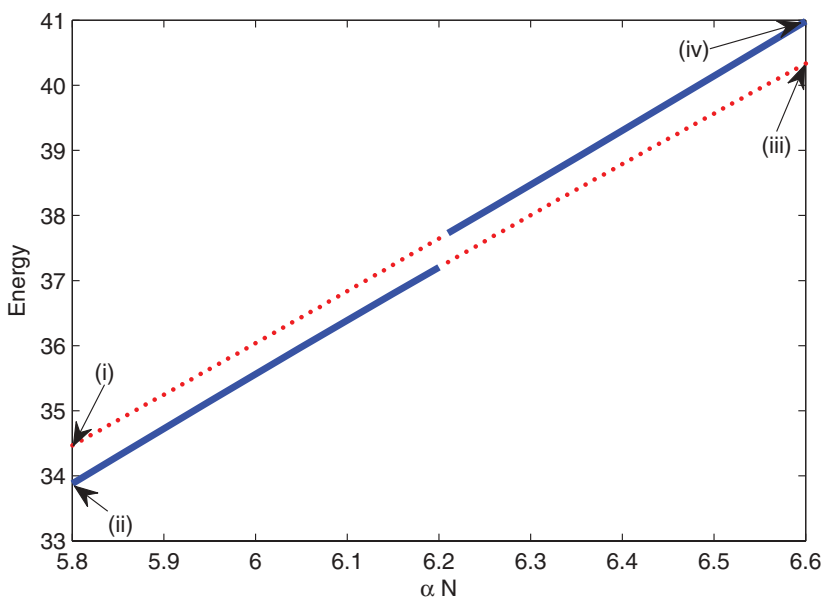

(i)

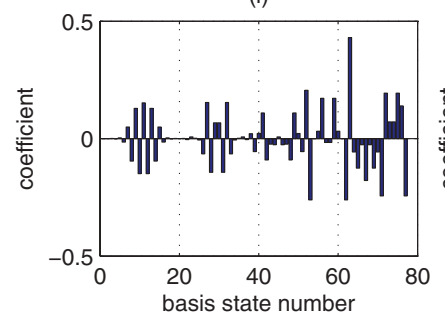

(iii)

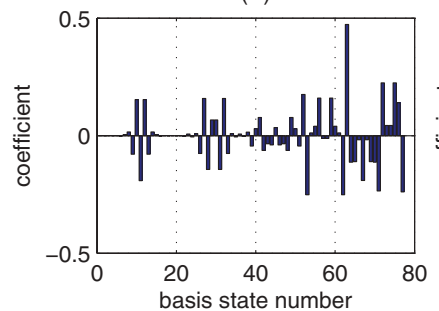

(ii)

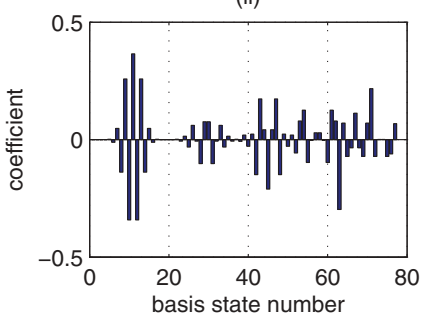

(iv)

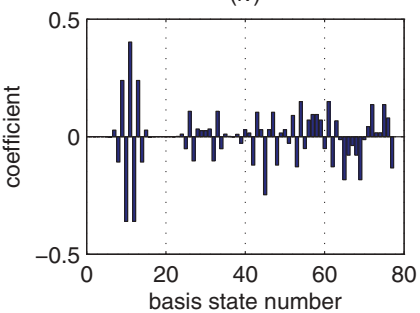

FIG. 2. (Color online) Left: Magnification of an avoided crossing corresponding to a strong resonance in Fig. 1 for $N=20$ particles. Right: comparison of the eigenstates on both sides of the crossing, at the points (i), (ii), (iii), and (iv) marked with arrows. In these figures, coefficients of the basis states (4) are arranged in the following order: $|0, N, 0\rangle_{0},|1, N-1,0\rangle_{0},|2, N-2,0\rangle_{0}, \ldots,|N-1,1,0\rangle_{0},|N-2,1,1\rangle_{0}, \mid N-$ $3,2,1\rangle_{0}, \ldots,|2, N-3,1\rangle_{0},|N-4,2,2\rangle_{0}, \ldots$ (Note that, for instance, $|1, N-2,1\rangle_{0}$ did not follow $|2, N-3,1\rangle_{0}$ since $|1, N-2,1\rangle_{0}$ is a translation of $|N-2,1,1\rangle_{0}$ which occurred earlier in the series.) State number 11 is thus $|10,10,0\rangle_{0}$, and all states with numbers larger than 20 correspond to nonzero population on all three sites.

When nonlinearity decreases, it is not a priori evident which of the quantum eigenstates to follow, since the $[N / 2, N / 2,0]$ state starts to mix with other states through avoided crossings. One intuitive suggestion is to identify the "right" quantum eigenstate as the state which has its largest expansion coefficient for the basis function $|N / 2, N / 2,0\rangle_{k}$. This may not uniquely identify an eigenstate; another property of a "good" eigenstate that should be checked is that the signs of the coefficients for basis functions $|N / 2 \pm 1, N / 2 \mp 1,0\rangle_{k}$ should be opposite to that of $|N / 2, N / 2,0\rangle_{k}$ (and there will be further sign alternations when more bosons tunnel between these two sites). This is true for the corresponding SU(3) coherent state, and can be intuitively understood since the antiphase oscillations of the two sites in the classical limit would require a boson to change the sign of its wave function to tunnel between these sites. One should check separately the two cases $k=0$ (bold curves in Fig. 1) and $k= \pm 2 \pi / 3$ (in the latter case it is more convenient to take the two linear combinations giving real states).

Since the classical unstable dynamics originates in the resonant oscillation between a mode essentially populating the empty site and another mode of internal population oscillations between the two nonzero sites [1], quantum mechanics must reproduce the corresponding oscillations for large $N$. Thus, one could expect that in the unstable regime, there should be a strong mixing (corresponding to avoided crossings) with states having large expansion coefficients for basis functions of the form $|N / 2 \pm p, N / 2 \mp p, 0\rangle_{k}$ and $|N / 2-q, N / 2-q, 2 q\rangle_{k}$, respectively, where $p$ and $q$ are some small integers corresponding to transfer of a few bosons from the " $[N / 2, N / 2,0]$ " state in the classical internal-mode directions. There are in general several such eigenstates which, in addition to the different values of the Bloch vector $k=0, \pm 2 \pi / 3$, also may be either symmetric or antisymmetric under site permutations when $k=0$ [15], and avoided crossings may appear only with those of the same symmetry as the $[N / 2, N / 2,0]$ state. An example of such an avoided crossing for $N=20$ is illustrated in Fig. 2. As can be seen for decreasing nonlinearity, the state with the largest expansion coefficient for the basis function $|N / 2, N / 2,0\rangle_{0}$ [bold blue line, states (ii) and (iv)] will pick up an additional peak at the crossing, which corresponds to the basis state (here number 63) where the crossing state [dotted red line, states (i) and (iii)] has its main peak. Here, the main resonant basis state is $|4,4,12\rangle_{0}$, and thus it can be viewed as corresponding to a transfer of $q=6$ bosons equally from the populated sites to the empty site. Similar analyses can be made for other main resonances. For example, for the case with $N=20$ particles in Fig. 1, we find five avoided crossings involving the $[N / 2, N / 2,0]$ state in the interval $5.5 \lesssim \alpha N \lesssim 10$, and in addition to the case shown in Fig. 2 major resonance peaks can be identified for basis functions $|11,8,1\rangle_{0},|12,7,1\rangle_{0},|9,9,2\rangle_{0},|9,8,3\rangle_{0},|12,5,3\rangle_{0}$.

Even though the above figures show that it is possible to identify a reasonably smooth curve in the spectrum (away from the strongest resonances) as a $\operatorname{good}[N / 2, N / 2,0]$ state, the properties of such eigenstates drastically change in a regime around the classical oscillatory instability. This is illustrated in Fig. 3, where the quantity $\left|c_{N / 2, N / 2,0}^{\max }\right|^{2} \equiv$ $\max _{\mathrm{ES}}\left|\left\langle\frac{N}{2}, \frac{N}{2}, 0 \mid \mathrm{ES}\right\rangle_{k}\right|^{2}$ is plotted as a function of $\alpha N$ for $N$ increasing from 10 to 80 particles (in the following, ES will be used to denote "eigenstate" in formulas and figures). It is evident that, as $N$ increases, this quantity drops considerably in the region that corresponds to the classically unstable regime, and thus, in any eigenstate, the probability to find the particles equally distributed on only two sites becomes very small in this regime. Thus, this shows a clear quantum signature of the classical oscillatory instability.

We can also observe that the avoided crossings in the spectrum (Fig. 2) typically appear in the region where the curves in Fig. 3 drop. The conclusion is therefore that the drop 

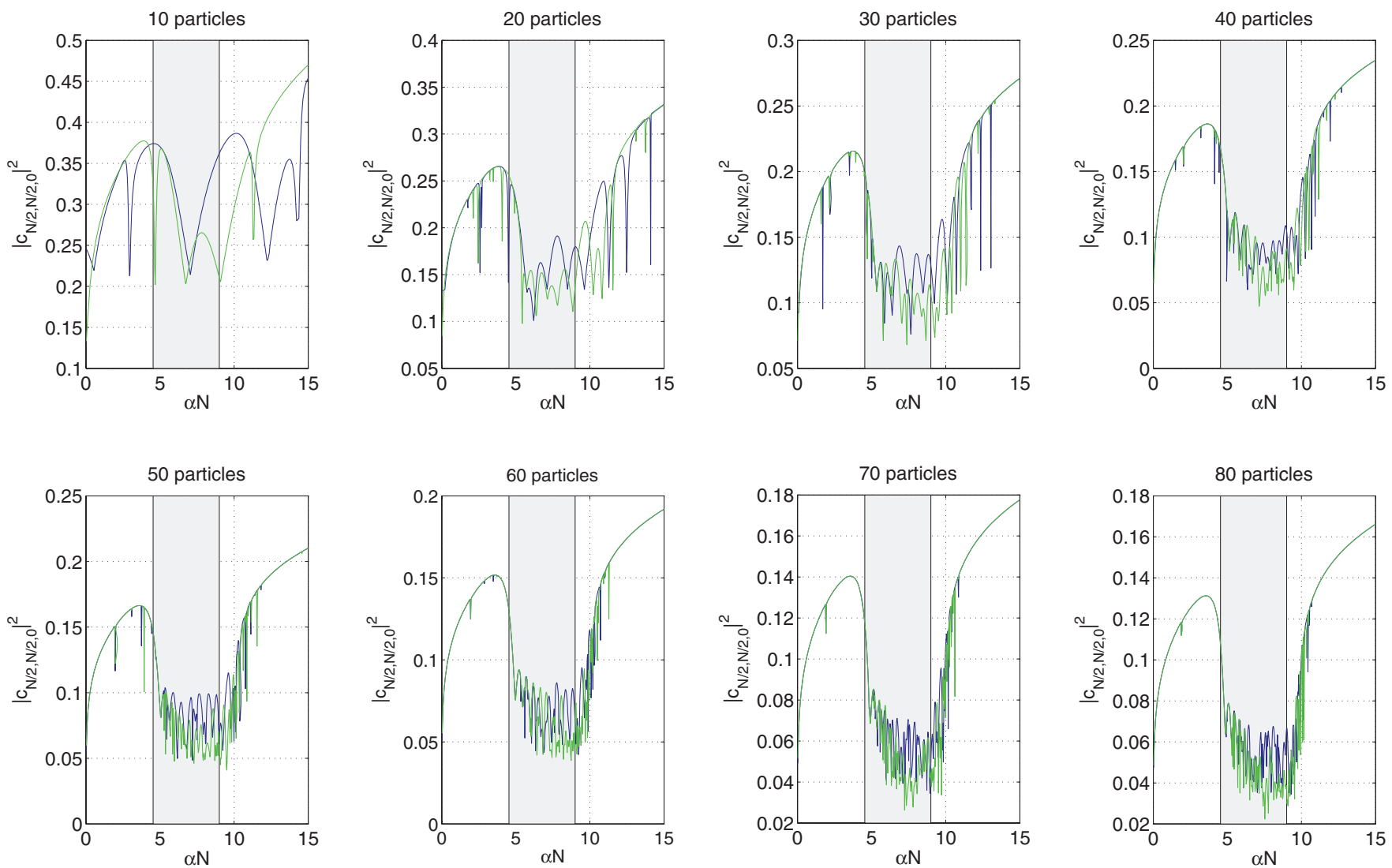

FIG. 3. (Color online) The maximum probability that, in any eigenstate, all particles are equally distributed between only two sites, plotted as a function of $\alpha N$ for $N$ between 10 and 80 particles. Blue (darker) and green (lighter) curves correspond to $k=0$ and $k= \pm 2 \pi / 3$, respectively. The shaded region marks the classical instability regime.

in Fig. 3, and also the instability, are related to the avoided crossings and the mixing of eigenstates. The avoided crossings that, possibly, occur outside this region cannot be seen in our plots, which means that the eigenstates would get very close and the mixing would therefore be very swift.

A complementary approach is to look globally rather than locally at the eigenspectrum, and consider a measure of the total overlap between symmetric "compact" SDW basis states $|N / 2, N / 2,0\rangle_{k}$ and basis states which are not two-site localized, as summed over all the eigenstates for a given $N$. This can be considered as a necessary (but generally not sufficient) condition for development of an instability: if the overlap is small, an initial exact zero in a SDW excitation can never grow large. The quantity that we use to measure this "overlap" property is

$\Upsilon \equiv \sum_{\mathrm{ES}}\left|\left\langle\frac{N}{2}, \frac{N}{2}, 0 \mid \mathrm{ES}\right\rangle_{k}\right|^{2}\left(1-\sum_{n=1}^{N-1}\left|\langle N-n, n, 0 \mid \mathrm{ES}\rangle_{k}\right|^{2}\right)$.

A plot of $\Upsilon$ is shown in Fig. 4. Low values of $\Upsilon$ implies that eigenstates with a large portion of $\left|\frac{N}{2}, \frac{N}{2}, 0\right\rangle_{k}$ contain very little of states not entirely located on two sites. They will therefore not excite the empty site much. As is clear from Fig. 4, a plateau of high values of $\Upsilon$ starts to develop as $N$ increases (a tendency can be seen already for $N=10$ bosons), and already for $N=80$ it corresponds almost perfectly to the classical instability regime. Thus, this measure can also be used as a clear instability indicator here. (The increase for small $\alpha$ is not related to any instability, but reflects the general delocalization tendency of eigenstates in the linear limit.)

\section{DYNAMICS}

A number of numerical simulations of the dynamics have been performed, in order to elucidate to what extent the quantum dynamics, for relatively small particle numbers, may reproduce essential features of the classical SDW state transition from stable internal-mode oscillations to oscillatory instability. Since there is no unique choice of quantum initial state corresponding to the classical SDW stationary state, we will here separately discuss two alternative possibilities: slightly perturbed specific eigenstates (Sec. IV A) and SU(3) coherent states (Sec. IV B).

\section{A. Eigenstate}

The analysis in Sec. III indicates that we can generally identify a "quantum SDW" eigenstate, interacting resonantly with symmetry-breaking eigenstates in the regime of classical instability. We may thus investigate the dynamics for a slightly perturbed quantum SDW eigenstate by making a small, symmetry-breaking change in some expansion coefficents, and check if the classical transition from stable oscillations 

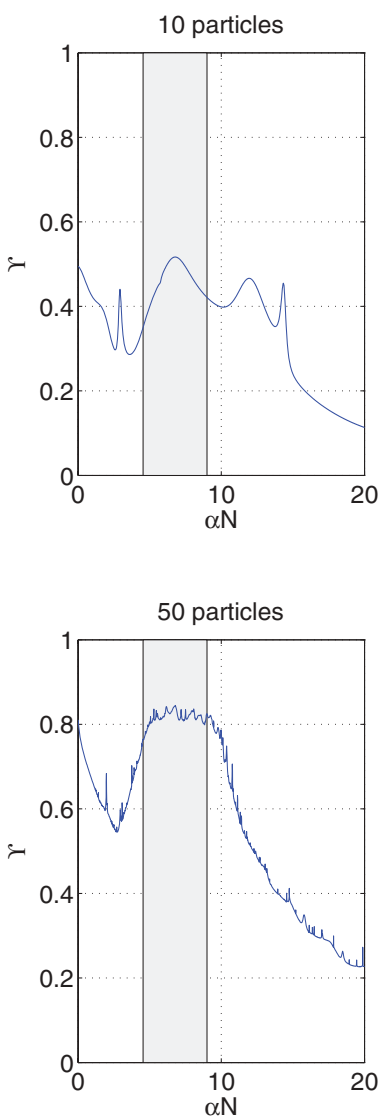
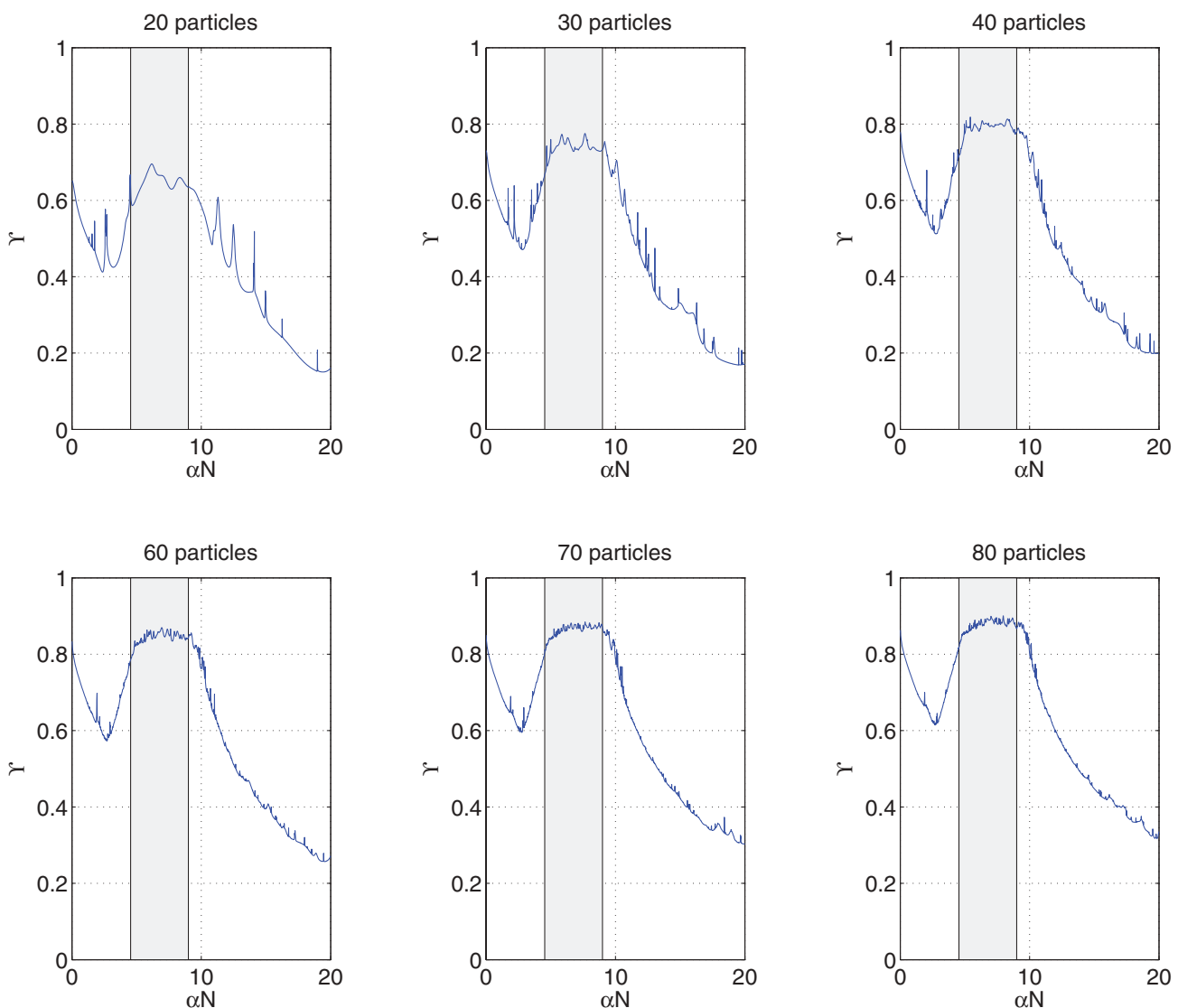

FIG. 4. (Color online) The quantity $\Upsilon$ (5), measuring total overlap in eigenstates between symmetric compact SDW components and components which are not two-site localized, plotted as a function of $\alpha N$ for $N$ between 10 and 80 particles. The shaded region marks the classical instability regime.

to unstable may be reproduced for sufficiently large $N$. It is important to consider all relevant quantum time scales, which must be separated in order to observe classical-like dynamics.

Since the eigenstates are divided into three different subspaces $(k=0, \pm 2 \pi / 3)$ making them translationally invariant, a classical-like initial SDW state must break this translational invariance by adding SDW-like eigenstates from all the subspaces. The energy splitting between these, $\Delta E_{k}$, then yields an oscillation period $T_{t}=2 \pi / \Delta E_{k}$ for tunneling of the SDW site, and if classical dynamics is to be reproduced, we must have $\Delta E_{k} \rightarrow 0$ as $N \rightarrow \infty$ for some specific "tunneling pair" [25-27] of nearly degenerate SDW-like eigenstates (strictly speaking there are of course three states involved, but states with $k= \pm 2 \pi / 3$ are always degenerate). Considering, as in Sec. III, the SDW-like eigenstates as eigenstates that maximize the $|N / 2, N / 2,0\rangle_{k}$ expansion coefficient, there is some ambiguity in how to choose an optimal tunneling pair, since the eigenstates with largest $|N / 2, N / 2,0\rangle_{k}$ coefficient may have different characters for $k=0$ and $k= \pm 2 \pi / 3$ in the instability regime, as the avoided crossings appear in different places (cf. Fig. 3). We here selected the eigenstate, in the subspace for $k=0$, with the largest coefficient for $|N / 2, N / 2,0\rangle_{0}$. We can then "project" this eigenstate on the eigenstates in the other subspaces if we introduce a pseudoscalar product defined by the property ${ }_{k^{\prime}}\left\langle n_{1}^{\prime}, n_{2}^{\prime}, n_{3}^{\prime} \mid n_{1}, n_{2}, n_{3}\right\rangle_{k}=\delta_{n_{1}^{\prime} n_{1}, n_{2}^{\prime} n_{2}, n_{3}^{\prime} n_{3}}$. This makes it possible to pick out eigenstates with $k= \pm 2 \pi / 3$ having the largest overlap with our selected eigenstate from $k=0$. The tunneling oscillation period $T_{t}$ resulting from this energy splitting is illustrated in Fig. 5. As is clearly seen, the oscillation period rapidly grows with particle number in the classically stable regimes (notice the logarithmic scales), but remains considerably smaller in the regime of classical instability. Thus, this indicates a fundamental problem in any attempt to reproduce features of the classical instability dynamics with initial conditions chosen from such quantum eigenstates: the quantum tunneling oscillations may occur on the same time scales as (or smaller than) the development of the instability, even for rather large particle numbers.

A good SDW-like state constructed from quantum eigenstates should also have certain other classical-like properties, for example, $\left\langle\mathrm{ES}\left|\hat{n}_{3} / N\right| \mathrm{ES}\right\rangle \approx 0$ and $\left\langle\mathrm{ES}\left|\hat{n}_{1} \hat{n}_{2} / N^{2}\right| \mathrm{ES}\right\rangle \approx 1 / 4$. To test this we can measure $\left\langle n_{i}\right\rangle$ for all eigenstates, after breaking the translational invariance by adding states from the different subspaces. The optimal SDW triplet of nearly degenerate states chosen as above are added together $(1 / \sqrt{3}$ of each eigenstate), and the phase of each eigenstate is selected so that the coefficient $c_{N / 2, N / 2,0}^{k}$ of $|N / 2, N / 2,0\rangle_{k}$ is real and positive. There is of course an ambiguity also in the choice of phases when we add the eigenstates, and in general it is possible that we do not access the state with optimal values of $\left\langle n_{i}\right\rangle$, i.e., we may have an intermediate state that has "tunneled halfway" between two sites. By demanding that $c_{N / 2, N / 2,0}^{k}$ is positive and real, this should be avoided. 

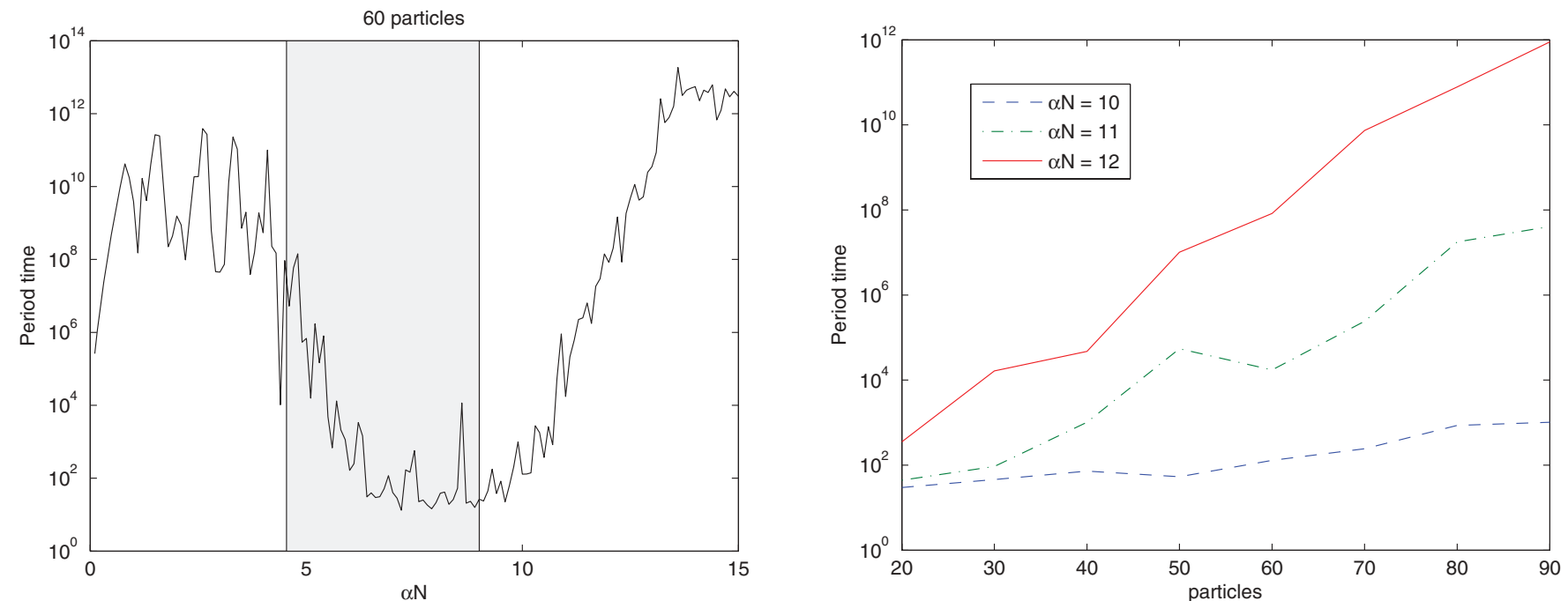

FIG. 5. (Color online) Oscillation period $T_{t}=2 \pi / \Delta E_{k}$ corrsponding to energy splitting between the eigenstate with maximum coefficient for the basis function $|N / 2, N / 2,0\rangle_{0}$ when $k=0$, and a corresponding eigenstate with $k=2 \pi / 3$ as described in the text vs (left) $\alpha N$ for fixed $N=60$ particles (the saturation around $10^{12}$ is due to using a limited numerical accuracy), and (right) particle number $N$ for fixed $\alpha N=10,11,12$ (from bottom to top). The shaded region in the left figure marks the classical instability regime.

In Fig. 6 we show $\left\langle n_{i} / N\right\rangle_{\min }$ and $\left\langle n_{i} n_{j} / N^{2}\right\rangle_{\max }$, respectively, for the states maximizing the $|N / 2, N / 2,0\rangle_{0}$ expansion coefficient, as a function of $\alpha N$ for different numbers of particles. From these plots it is evident that $\left\langle n_{i} / N\right\rangle_{\min }$ $\left(\left\langle n_{i} n_{j} / N^{2}\right\rangle_{\max }\right)$ is not a strictly decreasing (increasing) function of the number of particles in the unstable region. On the other hand, in the classically stable regimes they well approach the classically expected values $0(0.25)$ already for $N$ of the order of 100 particles. Note that Fig. 6 also shows an interesting feature in terms of plateaus developing in the upper part of the classical instability region. This might be a sign of the strongly chaotic regime, surrounding the classical unstable SDW solution for these parameter values [1].
Note also the different behaviors around the lower and upper classical instability thresholds: Around the lower threshold, where the classical unstable dynamics is self-trapped [1], the expectation values slowly increase (decrease) from their classical values until stronger resonances start to appear around the self-trapping transition at $\alpha N \approx 5.3$. On the other hand, at the upper threshold the expectation values remain nonclassical also in a part of the classically stable regime $(9<\alpha N \lesssim 10$ for $N=100$ ) where they only slowly approach the classical values as $N$ increases.

We also found other SDW-like candidates among the eigenstates in the classical instability regime, using the same criteria as above $\left(\left\langle\mathrm{ES}\left|\hat{n}_{3} / N\right| \mathrm{ES}\right\rangle \approx 0\right.$ and $\left.\left\langle\mathrm{ES}\left|\hat{n}_{1} \hat{n}_{2} / N^{2}\right| \mathrm{ES}\right\rangle \approx 1 / 4\right)$.
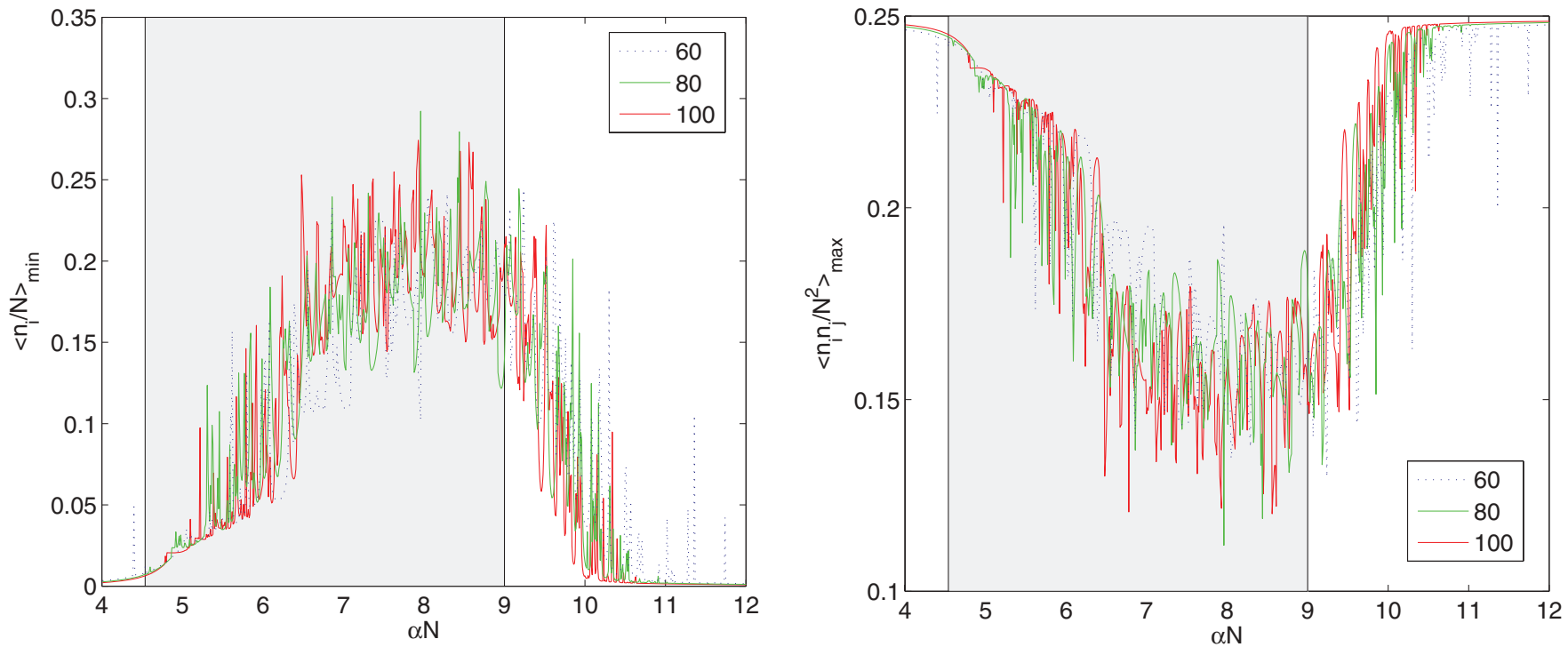

FIG. 6. (Color online) $\left\langle n_{i} / N\right\rangle_{\min }$ (left) and $\left\langle n_{i} n_{j} / N^{2}\right\rangle_{\max }$ (right) as a function of $\alpha N$, for linear combinations of eigenstates with $k=$ $0, \pm 2 \pi / 3$ with maximum coefficient for the basis function $|N / 2, N / 2,0\rangle_{0}$, for $N=60$ (dotted blue), $N=80$ (light green), and $N=100$ (dark red), particles. The shaded region marks the classical instability regime. 

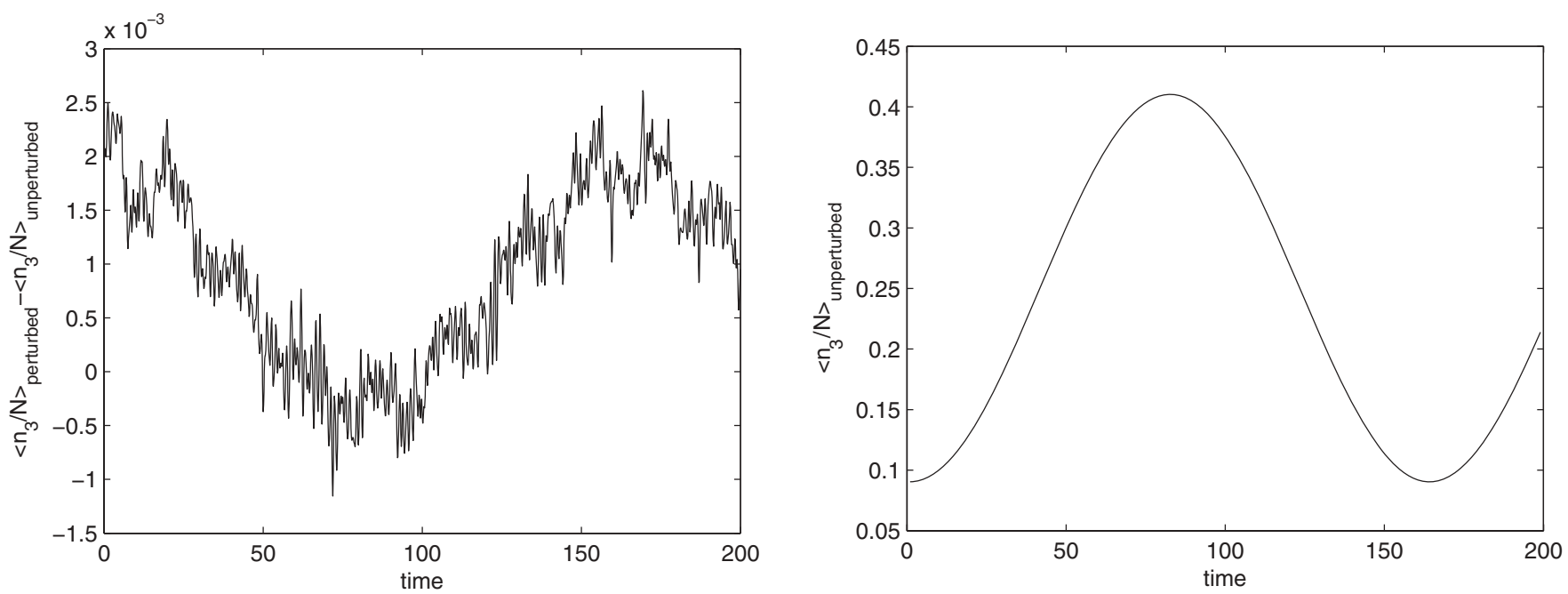

FIG. 7. Left: Difference $\Delta\left\langle n_{3} / N\right\rangle$ in occupation of site 3 (having initially smallest $\left\langle n_{i}\right\rangle$ ) between an exact and a perturbed tunneling pair of eigenstates, prepared as described in text. Right: Dynamics over the same time range of $\left\langle n_{3} / N\right\rangle$ for the unperturbed tunneling pair. $\alpha N=6$, $N=60$.

However, these states did not show the expected antisymmetric structure (tunneling of one particle connected with a minus sign), while the eigenstate that maximizes the $|N / 2, N / 2,0\rangle_{0}$ coefficient generally does (see the examples in Fig. 2, and also in Fig. 9 below).
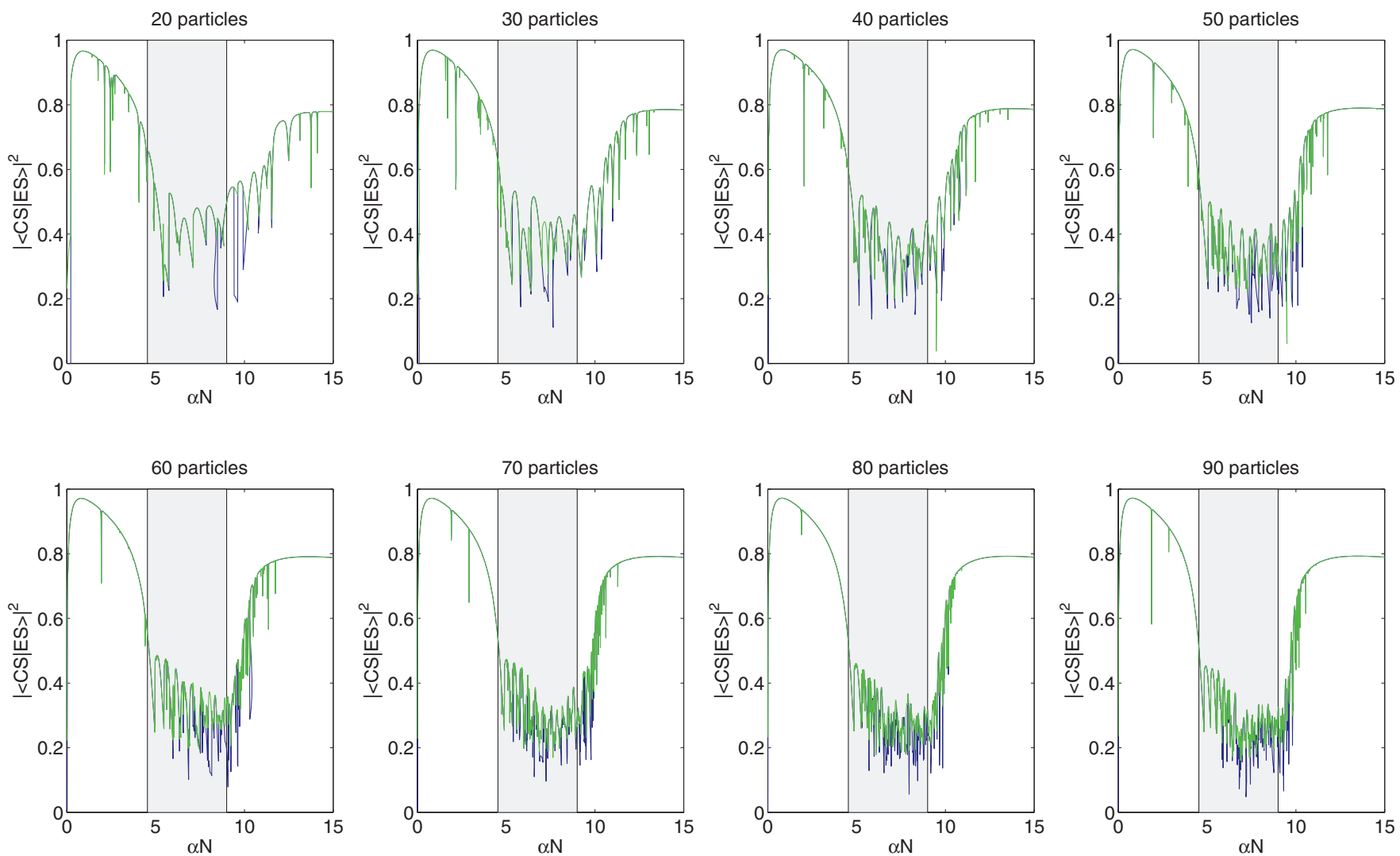

FIG. 8. (Color online) Projection of SU(3) coherent states onto tunneling pairs of eigenstates with maximal expansion coefficient for $|N / 2, N / 2,0\rangle_{0}$. Blue (dark) curves, eigenstates chosen as in Sec. IV A; green (light) curves, linear combinations of eigenstates with maximum coefficients for $|N / 2, N / 2,0\rangle_{k}$ for each $k$. [The blue (dark) curves coincide with the green (light) curves in regimes where only the latter can be seen.] The shaded region marks the classical instability regime. 

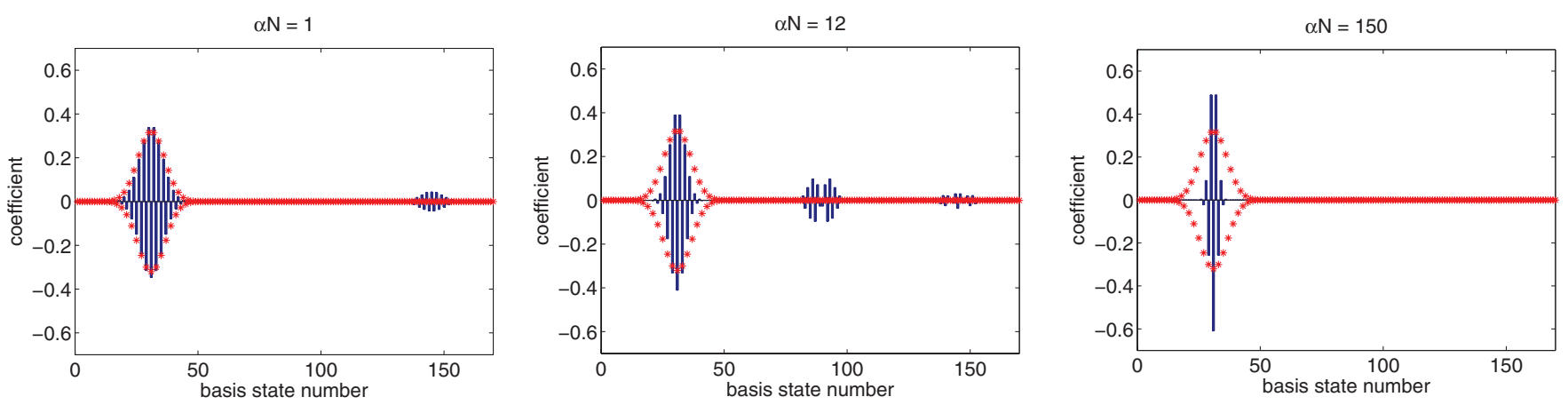

FIG. 9. (Color online) Coefficients of dominating Fock basis states for SDW SU(3) coherent states (red crosses) and for the $k=0$ eigenstate with largest projection on the SU(3) coherent state (blue bars) when $N=60$ and (left) $\alpha N=1$; (middle) $\alpha N=12$; (right) $\alpha N=150$. The ordering of the basis states is the same as in Fig. 2, and thus state number 31 is $|30,30,0\rangle_{0}$, and all states with numbers higher than 60 correspond to nonzero population at all three sites.

coefficients of $\left|n_{1}, n_{2}, n_{3}\right\rangle_{k}$. A typical example of the resulting dynamics in the classically unstable regime is shown in Fig. 7. As can be seen, in contrast to the classically perturbed unstable SDW dynamics the difference of $\left\langle n_{3}\right\rangle$ between the perturbed and unperturbed states does not grow at all, and thus the dynamics of the perturbed eigenstate is not analogous to the classically perturbed dynamics. There is, however, an oscillation of $\left\langle n_{3}\right\rangle$ over longer time scales that can be attributed to the tunneling time scale $\Delta E_{k}$ discussed above, but this oscillation is essentially the same for the perturbed and unperturbed states, leaving only minor traces in the dynamics for $\Delta\left\langle n_{3} / N\right\rangle$.

The failure of a single tunneling pair of eigenstates to capture the essential features of the classical dynamics in the instability regime is related to the many avoided crossings, leading to a mixing between the chosen state and other eigenstates [cf. state (ii) in Fig. 2, which in addition to its main SDW-like peak in the left part contains large non-SDW-
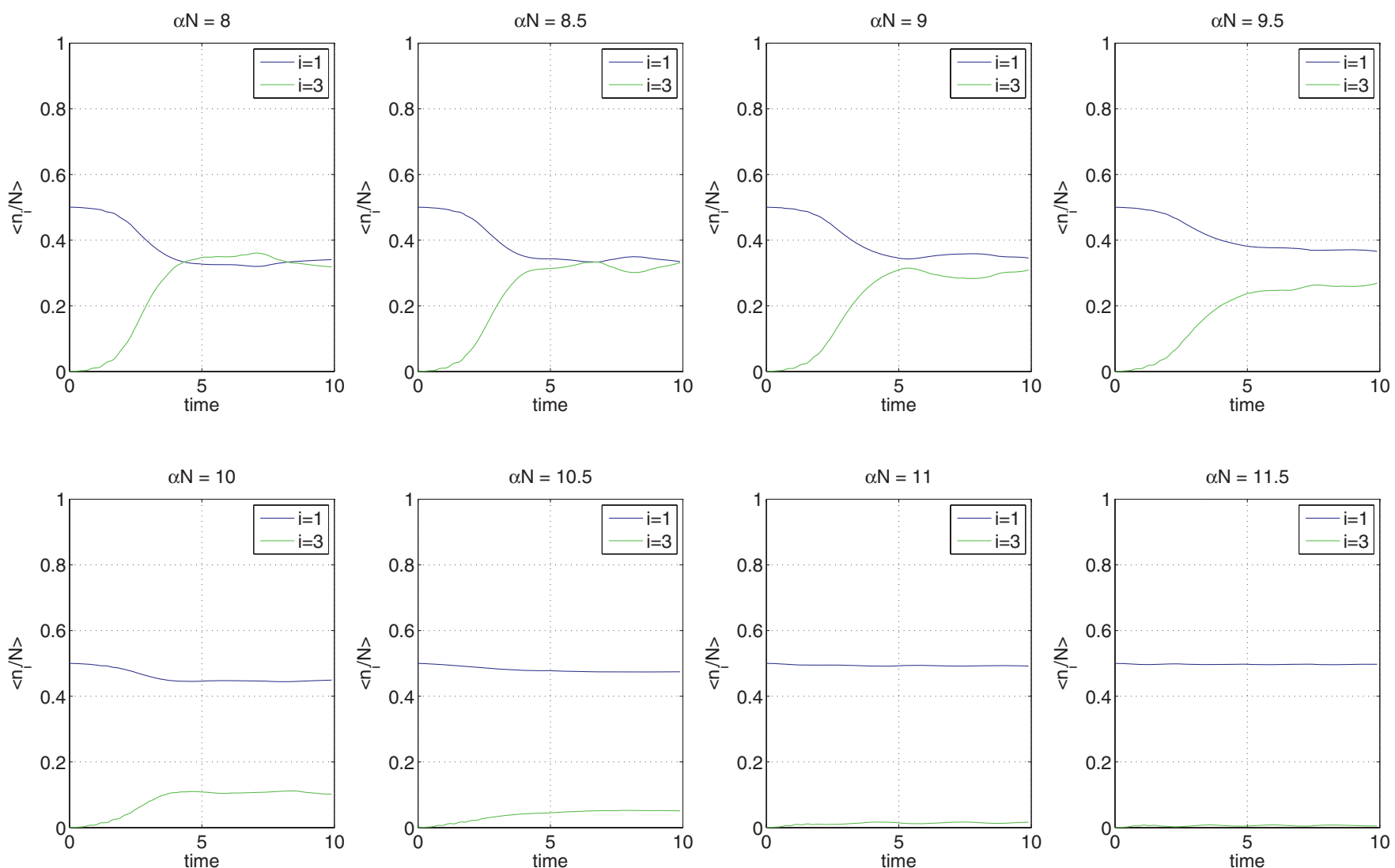

FIG. 10. (Color online) Short-time dynamics with SDW SU(3) coherent state as initial condition, for $N=80$ particles and $\alpha N$ varying from 8 to 11.5 (i.e., crossing the classical upper stability transition). Green lines (lower at $t=0$ ) show relative population expectation values $\left\langle n_{i} / N\right\rangle$ for the initially empty site, and blue lines (upper at $t=0$ ) for one of the two initially excited sites. 
like components in its tail in the right part]. Thus, in the instability regime already the unperturbed quantum state is too far away from a classical SDW state to reproduce the classical instability development [e.g., $\left\langle n_{3} / N\right\rangle$ is not even initially close to zero as seen from Figs. 6 and 7 (right)]. It is therefore reasonable to assume that the SDW-like properties are spread out over several eigenstates and that a "satisfying" classical-like SDW state can only be created by a superposition of many eigenstates. On the other hand, in the classically stable regimes where the expectation values in Fig. 6 are close to the classical values, the single tunneling pair well reproduces the classical dynamics with superposed tunneling oscillations with periods as in Fig. 5 (cf. Fig. 13, right part, below).

\section{B. Coherent state}

An SU(3) coherent state, converging to a classical SDW stationary solution in the classical limit, can be constructed as described, e.g., in [12-14,16,17]. In particular, we may use the explicit expansion in Fock eigenstates given in Eq. (2.16) in [14] with coherent state parameters $w_{1}=-1, w_{2}=0$ to construct, for any given particle number $N$, an $\mathrm{SU}(3)$ coherent state with exactly zero population on site 2, corresponding to a classical SDW state for $N \rightarrow \infty$.

These SU(3) coherent states are generally not eigenstates for finite $N$, and to compare with results from the previous section we plot in Fig. 8 [blue (dark) curves] the projection of the coherent state on the linear combination of the three nearly degenerate eigenstates from $k=0, \pm 2 \pi / 3$ with the largest $|N / 2, N / 2,0\rangle_{0}$ component (the eigenstate of Fig. 3), chosen
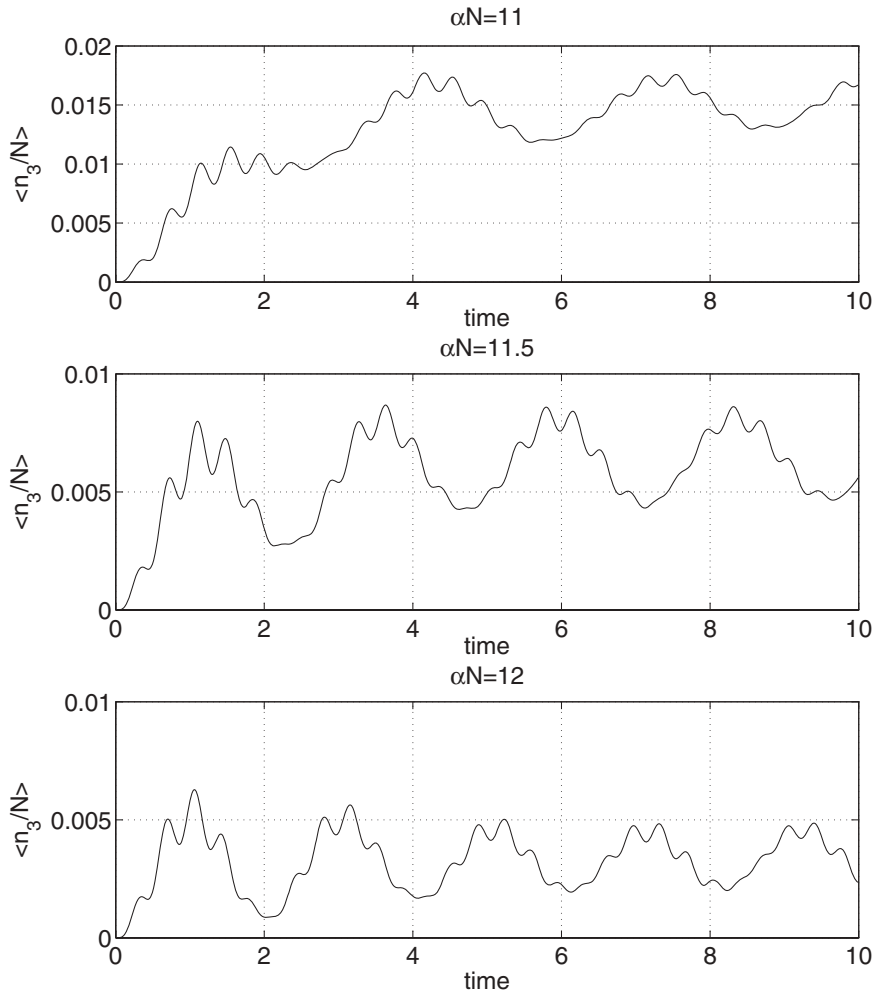

as described in Sec. IV A. We can see that the coherent state does not approach the eigenstates closely, especially not in the unstable region where the projection develops a pronounced $\operatorname{dip}$ as $N$ increases. We should note that the coherent state that we use is strictly zero on the "empty" site, while the eigenstates generally have probabilities for nonzero but small population also on this site, which becomes negligible in the classical limit. Figure 9 shows explicit comparisons between coherent states and the $k=0$ eigenstates with highest projection on the coherent state (which are precisely the states with largest coefficient for the $|N / 2, N / 2,0\rangle_{0}$ basis state) in regimes of classical stability. Note that for small $\alpha N$, the population of the third site is the main source of discrepancy between the coherent state and the eigenstate, while for large $\alpha N$ the main discrepancy occurs because the eigenstate narrows towards a single peak at $|N / 2, N / 2,0\rangle$ when $\alpha \rightarrow \infty$ for fixed $N$, while the width of the coherent state by definition remains unchanged. [A similar comparison between SU(3) coherent states and exact eigenstates was discussed for the ground state of the Bose-Hubbard trimer in [28].]

For comparison, we also show in Fig. 8 [green (light) curves] the corresponding projection of coherent states onto a linear combination of three eigenstates where we instead chose independently, for each $k$, the eigenstates with maximum coefficients for the basis state $|N / 2, N / 2,0\rangle_{k}$. As can be seen, in the classically stable regimes the curves coincide (the chosen eigenstates for $k= \pm 2 \pi / 3$ are then generally the same), while in the classically unstable regime the green (light) curves typically show less pronounced dips. The more pronounced dips for the blue (dark) curves are associated with the fact that,
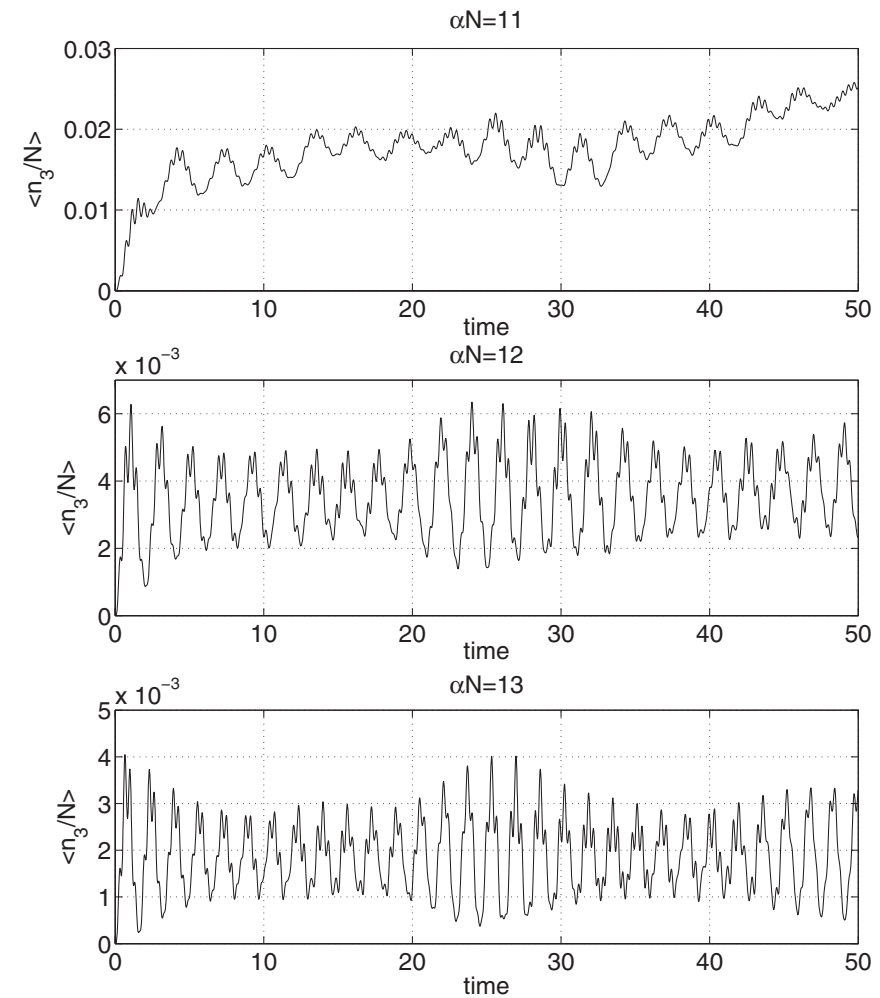

FIG. 11. Early dynamics showing the expectation value for the relative population of the initially unoccupied site, for a coherent-state initial condition with $N=80$ particles. Left: dynamics for $0<t<10$ and, from top to bottom, $\alpha N=11,11.5,12$; right: dynamics for $0<t<50$ and, from top to bottom, $\alpha N=11,12,13$. 
close to strong resonances, the maximum overlap criterion may select states with $k= \pm 2 \pi / 3$ whose large overlap with the chosen $k=0$ state is not primarily due to the coherent part corresponding to zero population on the third site, but rather due to a strong incoherent tail of basis states with nonzero population on site 3 (cf. the examples in Fig. 2). It is also interesting to note that this feature becomes particularly pronounced in a regime of $\alpha N$ between approximately 5.3 and 9 , where the classical unstable SDW solution is surrounded by strong chaotic dynamics. On the other hand, for $N$ of the order of 90 particles, we see that the green (light) and blue (dark) curves in Fig. 8 essentially coincide for $4.5 \lesssim \alpha N \lesssim 5.3$, where the classical SDW solution is unstable but surrounded by KAM tori and therefore self-trapped (cf. Figs. 10 and 11 of [1]).

Figure 10 shows the short-time dynamics for a system with 80 particles when the coherent state is used as the initial state. We can see that, in certain aspects, it behaves similarly to a perturbed SDW stationary state from the classical dynamics, and in particular the transition from a self-trapped SDW dynamics in the stable regime to population mixing in the unstable is clearly seen as $\alpha N$ decreases. During a short time period in the beginning we can observe small-amplitude oscillations in both the stable and the unstable regions with frequencies that agree with the internal-mode oscillations of the classical dynamics [see, e.g., Eq. (12) and Fig. 1 in Ref. [1]]. This is illustrated more clearly in Fig. 11. Since the classical SDW solution in the stable regime has two internal oscillation modes for the amplitudes $a_{i}$, for a generic small perturbation the population $\left\langle n_{3}\right\rangle=\left|a_{3}\right|^{2}$ of the initially unexcited site should exhibit oscillations with the sum as well as the difference of these frequencies. Taking, e.g., the explicit example $\alpha N=12$ in Fig. 11 and plugging in the relevant numbers in the classical formula (12) of Ref. [1], we obtain oscillation periods $2 \pi /\left(\omega_{+}+\omega_{-}\right) \approx 0.34$ and $2 \pi /\left(\omega_{+}-\omega_{-}\right) \approx 1.9$, which agree well with the two major oscillations seen in Fig. 11. As $\alpha N$ decreases towards the instability transition the two classical frequencies approach each other, so that the larger oscillation period $2 \pi /\left(\omega_{+}-\omega_{-}\right)$ increases and diverges at the transition point. This tendency is also seen in Fig. 11. Notice also from the right figures in Fig. 11 that there is an additional modulation with period approximately 25 . This oscillation is a pure quantum feature with no classical analog; the period of this oscillation increases with $N$ (e.g., for $N=60$ we observe similar oscillations for $\alpha N=13$ but with a period of approximately 19).

It is worth observing that there is a gradual transition from the stable to unstable dynamics in Fig. 10, which is essentially a quantum effect since the classical transition occurs abruptly at the Hamiltonian Hopf bifurcation at $N=9$ [1,9]. In Fig. 12 we can see that for $\alpha N=10.0$ (classically stable regime) we get a dynamics that approaches the classical stable dynamics when we increase the particle number.

Finally, we give in Fig. 13 a comparison between the long-time dynamics in the classically stable regime with initial conditions given by an $\mathrm{SU}(3)$ coherent state and a
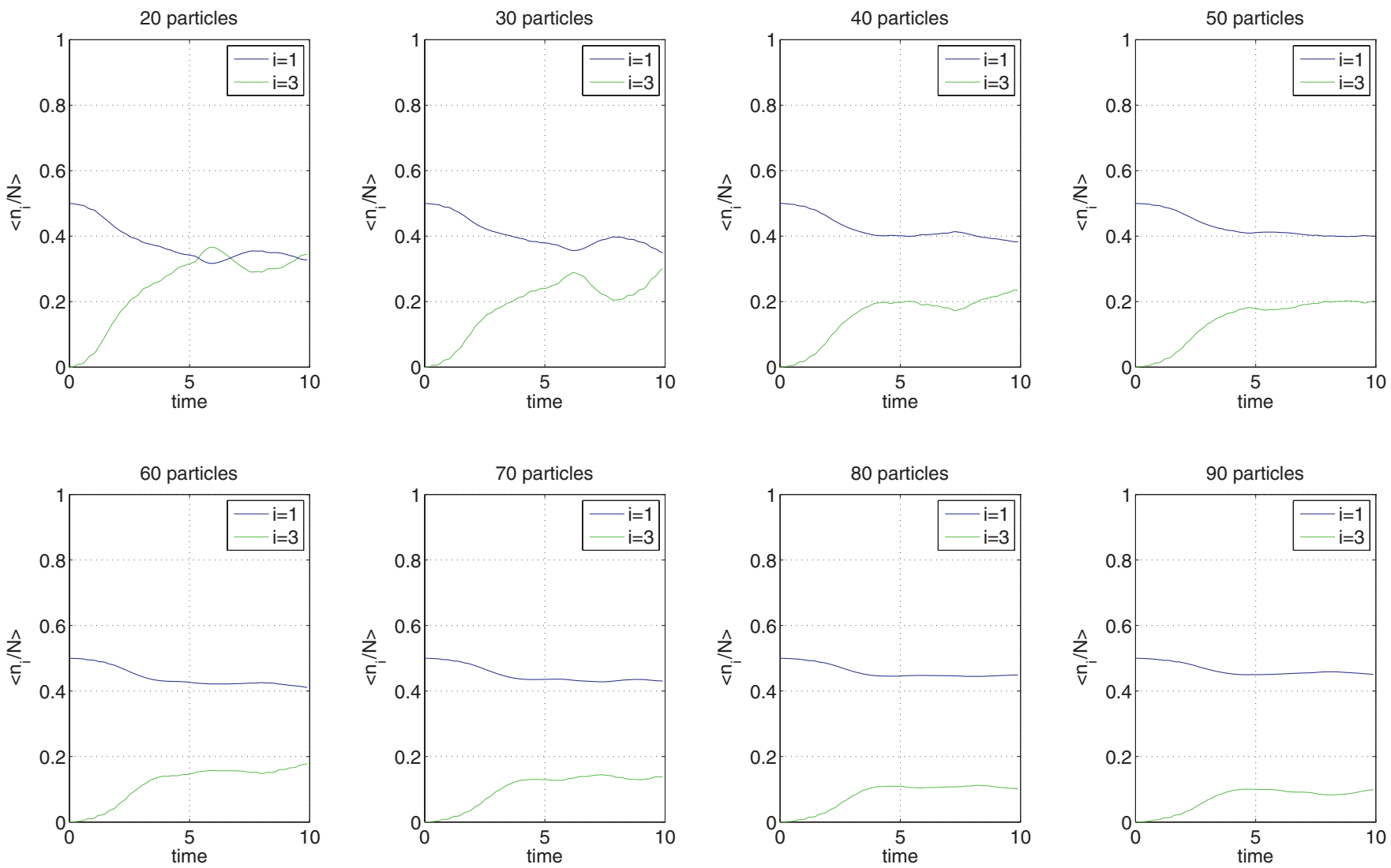

FIG. 12. (Color online) Dynamics for the coherent-state initial condition for 20-90 particles, respectively, for $\alpha N=10$. The same quantities as in Fig. 10 are plotted. 

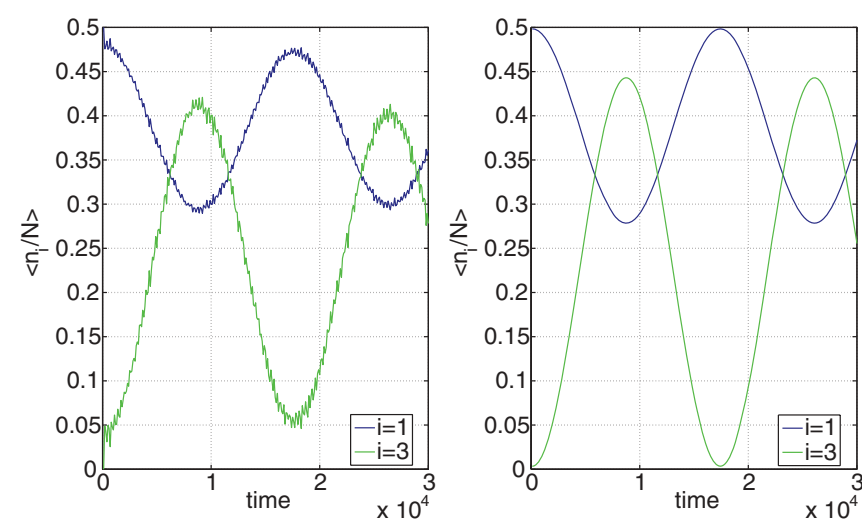

FIG. 13. (Color online) Long-time dynamics for a system of 60 particles and $\alpha N=11$, for initial conditions given by (left) the $\mathrm{SU}(3)$ coherent state, and (right) a linear combination of exact eigenstates constituting a SDW tunneling pair as in Fig. 5, prepared to minimize $\left\langle n_{3}(t=0)\right\rangle$ (pseudoeigenstate).

symmetry-broken SDW "pseudoeigenstate" prepared as a linear combination of $k=0$ and $k= \pm 2 \pi / 3$ exact SDW eigenstates as described in Sec. IV A, respectively. In both cases, the observed oscillation of the population expectation values between the sites correspond well to an oscillation period of the order $10^{4}$, as expected from tunneling oscillations (see Fig. 5). Note that, in these oscillations, the populations at sites 1 and 2 never drop to zero. Due to the symmetry of the initial condition, the inital hole at site 3 can only "split" symmetrically between sites 1 and 2, and after a full oscillation period recombine again at the original site 3 . Note that, while the symmetry-broken eigenstate initially has a small but nonzero value of $\left\langle n_{3} / N\right\rangle$ (cf. Figs. 6 and 9) which it returns to almost perfectly after one oscillation period, the coherent state has $\left\langle n_{3}\right\rangle=0$ exactly at $t=0$, but returns to a value significantly larger than zero after one period. This, together with the small superposed oscillations for the coherent state, are the main qualitative differences between the long-time dynamics of these two types of eigenstate in the classically stable regime.

\section{CONCLUSIONS}

In conclusion, using the Bose-Hubbard model for a triangular configuration, we have identified a number of quantum signatures of the classical oscillatory instability regime for the single-depleted-well stationary solution of the corresponding discrete nonlinear Schrödinger equation. Focusing on a transitional regime between strongly quantum and semiclassical behavior with particle numbers between 10 and 100, some major conclusions can be drawn:

(i) The regime of classical oscillatory instability is directly related to avoided crossings in the energy spectrum, and a strong mixing between a pure SDW eigenstate and other eigenstates corresponding to resonances which populate the originally unoccupied site as well as breaking the originally symmetric population of the occupied sites.

(ii) As a consequence of these resonances, we may construct several measures that give clear signatures of the classically unstable regime already for particle numbers of the order of 20-30. As a particular example, we showed that the maximum probability, in any eigenstate, to have particles equally distributed between only two sites drastically decreases in the classically unstable regime. As another measure, we calculated the total overlap (5) between compact SDW basis states and basis states which are not two-site localized, summed over all eigenstates, and found a pronounced plateau developing in the classically unstable regime.

(iii) While in the classically stable regimes a single tunneling pair of quantum eigenstates with $k=0, \pm 2 \pi / 3$ can be identified as corresponding to a classical SDW stationary state, attempts at a similar identification in the classically unstable regime fail to capture essential features of the classical dynamics. For example, we showed that the dynamics resulting from a slightly perturbed single tunneling pair of quantum eigenstates in the unstable regime cannot reproduce the development of the oscillatory instability. Thus, the classical unstable dynamics must be viewed as a consequence of global properties of the eigenstates, rather than of individual eigenstates.

(iv) Using instead SDW SU(3) coherent states as initial conditions for the dynamics, several features of the classical transition from stable internal oscillations to oscillatory instability could be reproduced. However, while the classical transition appears abruptly at a given bifurcation point, the quantum transition appears gradual. Close to the upper bifurcation point on the stable side, the quantum dynamics for small particle numbers may signal instability with a large population developing on the initially unoccupied site, but for increasing particle numbers the classically stable dynamics is seen to be recovered.

Thus, there are a number of interesting features related to the quantum dynamics of SDW states and their stabilityinstability transitions, and we hope that the signatures that we have described here may be experimentally observable for triple-well Bose-Einstein condensate configurations in the near future. We should stress that oscillatory instabilities also appear for many other types of classical stationary states in the DNLS model [1], and it would be interesting to investigate to what extent the specific quantum signatures that we have described here for the SDW states are generic.

\section{ACKNOWLEDGMENTS}

M.J. is grateful to J. C. Eilbeck for being an initial source of inspiration for this work. Financial support from the Swedish Research Council and the Swedish Institute is gratefully acknowledged.
[1] M. Johansson, J. Phys. A 37, 2201 (2004).

[2] J. L. Marín, S. Aubry, and L. M. Floría, Physica D 113, 283 (1998).
[3] S. Darmanyan, A. Kobyakov, and F. Lederer, JETP 86, 682 (1998).

[4] M. Johansson and Yu. S. Kivshar, Phys. Rev. Lett. 82, 85 (1999). 
[5] A. Alvarez, J. F. R. Archilla, J. Cuevas, and F. R. Romero, New. J. Phys. 4, 72 (2002).

[6] A. M. Morgante, M. Johansson, G. Kopidakis, and S. Aubry, Phys. Rev. Lett. 85, 550 (2000); Physica D 162, 53 (2002); M. Johansson, A. M. Morgante, S. Aubry, and G. Kopidakis, Eur. Phys. J. B 29, 279 (2002).

[7] A. V. Gorbach and M. Johansson, Phys. Rev. E 67, 066608 (2003); Eur. Phys. J. D 29, 77 (2004).

[8] L. Kroon, M. Johansson, A. S. Kovalev, and E. Yu. Malyuta, Physica D 239, 269 (2010).

[9] J. Carr and J. C. Eilbeck, Phys. Lett. A 109, 201 (1985); J. C. Eilbeck, P. S. Lomdahl, and A. C. Scott, Physica D 16, 318 (1985).

[10] R. Franzosi and V. Penna, Phys. Rev. E 67, 046227 (2003); P. Buonsante, R. Franzosi, and V. Penna, Laser Phys. 13, 537 (2003).

[11] J. C. Eilbeck, in Localization and Energy Transfer in Nonlinear Systems, edited by L. Vázquez, R. S. MacKay, and M. P. Zorzano (World Scientific, Hackensack, NJ, 2003), p. 177.

[12] P. Buonsante, V. Penna, and A. Vezzani, Phys. Rev. A 72, 043620 (2005).

[13] C. Lee, T. J. Alexander, and Yu. S. Kivshar, Phys. Rev. Lett. 97, 180408 (2006).

[14] T. F. Viscondi and K. Furuya, J. Phys. A 44, 175301 (2011).

[15] S. De Filippo, M. Fusco Girard, and M. Salerno, Nonlinearity 2, 477 (1989).
[16] K. Nemoto, C. A. Holmes, G. J. Milburn, and W. J. Munro, Phys. Rev. A 63, 013604 (2000).

[17] F. Trimborn, D. Witthaut, and H. J. Korsch, Phys. Rev. A 79, 013608 (2009).

[18] E. Wright, J. C. Eilbeck, M. H. Hays, P. D. Miller, and A. C. Scott, Physica D 69, 18 (1993).

[19] D. Ellinas, M. Johansson, and P. L. Christiansen, Physica D 134, 126 (1999).

[20] L. Amico and V. Penna, Phys. Rev. Lett. 80, 2189 (1998).

[21] Yu. S. Kivshar and M. Peyrard, Phys. Rev. A 46, 3198 (1992).

[22] E. Altman, A. Polkovnikov, E. Demler, B. I. Halperin, and M. D. Lukin, Phys. Rev. Lett. 95, 020402 (2005); A. Polkovnikov, E. Altman, E. Demler, B. I. Halperin, and M. D. Lukin, Phys. Rev. A 71, 063613 (2005).

[23] L. Bernstein, Physica D 68, 174 (1993).

[24] P. Buonsante, R. Burioni, E. Vescovi, and A. Vezzani, Phys. Rev. A 85, 043625 (2012).

[25] L. Bernstein, J. C. Eilbeck, and A. C. Scott, Nonlinearity 3, 293 (1990).

[26] R. A. Pinto and S. Flach, Phys. Rev. A 73, 022717 (2006).

[27] R. A. Pinto and S. Flach, in Dynamical Tunneling: Theory and Experiment, edited by S. Keshavamurthy and P. Schlagheck (Taylor \& Francis, London, 2011), Chap. 14.

[28] P. Buonsante, V. Penna, and A. Vezzani, Phys. Rev. A 82, 043615 (2010). 